\title{
The transcriptome of Leishmania major in the axenic promastigote stage: transcript annotation and relative expression levels by RNA-seq
}

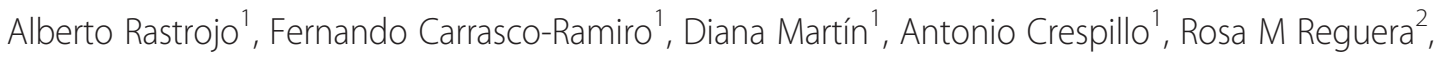 \\ Begoña Aguado ${ }^{1^{*}}$ and Jose M Requena ${ }^{1 *}$
}

\begin{abstract}
Background: Although the genome sequence of the protozoan parasite Leishmania major was determined several years ago, the knowledge of its transcriptome was incomplete, both regarding the real number of genes and their primary structure.

Results: Here, we describe the first comprehensive transcriptome analysis of a parasite from the genus Leishmania. Using high-throughput RNA sequencing (RNA-seq), a total of 10285 transcripts were identified, of which 1884 were considered novel, as they did not match previously annotated genes. In addition, our data indicate that current annotations should be modified for many of the genes. The detailed analysis of the transcript processing sites revealed extensive heterogeneity in the spliced leader (SL) and polyadenylation addition sites. As a result, around $50 \%$ of the genes presented multiple transcripts differing in the length of the UTRs, sometimes in the order of hundreds of nucleotides. This transcript heterogeneity could provide an additional source for regulation as the different sizes of UTRs could modify RNA stability and/or influence the efficiency of RNA translation. In addition, for the first time for the Leishmania major promastigote stage, we are providing relative expression transcript levels.
\end{abstract}

Conclusions: This study provides a concise view of the global transcriptome of the L. major promastigote stage, providing the basis for future comparative analysis with other development stages or other Leishmania species.

Keywords: Gene Expression, RNA-seq, Transcript annotation, mRNAs, Leishmania, Trypanosomatids

\section{Background}

Species of the genus Leishmania are protozoan parasites and aetiological agents of a spectrum of clinical diseases, known as leishmaniases, ranging from disfiguring skin lesions to life-threatening visceral infection. The World Health Organization (WHO) estimates that 350 million people worldwide are at risk of infection, and this disease is considered a major public health problem. Two million new cases of leishmaniasis (1.5 million for cutaneous forms and 500000 for visceral leishmaniasis) occur annually [1]. The genus Leishmania belongs to the order Trypanosomatida [2], which also includes, among others, Trypanosoma brucei and Trypanosoma cruzi, causative

\footnotetext{
*Correspondence: baguado@cbm.uam.es; jmrequena@cbm.uam.es 'Centro de Biología Molecular "Severo Ochoa" (CSIC-UAM), Universidad Autónoma de Madrid, 28049 Madrid, Spain

Full list of author information is available at the end of the article
}

agents of two other important human infectious diseases: sleeping sickness and Chagas disease, respectively. The evolutionary origin of these organisms is found in the deepest roots of the eukaryotic tree [3], and are characterized by markedly original molecular features.

In 1999, the complete sequence of chromosome 1 of Leishmania major was published and showed a remarkable feature of the gene organization in Leishmania, i.e. genes are grouped in large clusters sharing the same transcriptional direction. Thus, from the left end of chromosome 1, the first 29 genes are all located on the same DNA strand, whereas the remaining 50 genes are located on the other strand [4]. When transcriptional activity was examined by nuclear run-on analyses using single-stranded DNA probes, the protein-coding strand was found to be more strongly transcribed than the non-coding strand in the majority of the chromosome 1

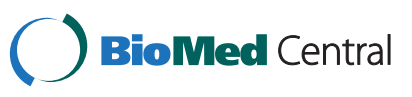


genes [5]. Furthermore, it was found that the RNA polymerase initiates transcription within the strand-switch region of chromosome 1. Similarly, in chromosome 3, which contains two convergent clusters of 67 and 30 genes, nuclear run-on analyses indicated that transcription initiates upstream of the most-5' gene of the two long polycistronic clusters [6]. After whole genome sequences for Leishmania and other trypanosomatids (i.e. T. brucei and T. cruzi) were completed, it was confirmed that in these organisms most genes are organized into large clusters on the same DNA strand.

Another remarkable molecular feature found in trypanosomatids is that transcription initiation by RNA polymerase II (RNAP II) is not regulated on a per gene basis; instead, most genes are transcribed polycistronically. Genome-wide chromatin immunoprecipitation analysis of L. major promastigotes showed acetylated histone H3 peaks at the $5^{\prime}$ ends of all polycistronic protein-coding gene clusters, indicating that global regulation of transcription initiation may be achieved by epigenetic regulation of $\mathrm{H} 3$ acetylation at the origins of polycistronic transcription units [7]. In a recent publication, the J base (a modification of thymine, which is introduced with some frequency in the DNA of trypanosomatids) was shown to define the RNAP II transcription termination sites in L. major and L. tarentolae [8].

In contrast to operons in bacteria, polycistronic units in trypanosomatids require processing before translation, and the mature mRNAs are processed from primary transcripts by coupled trans-splicing and polyadenylation [9]. During trans-splicing, a conserved spliced leader RNA (SL RNA or mini-exon) is added to the 5' end of all mRNAs, providing the cap structure for translation. The differential expression of mature mRNAs from a single polycistronic unit is thought to be achieved by post-transcriptional control, i.e. mRNA levels are regulated by RNA stability and/ or differential translation [10-12].

In 2005, the sequence for the 36 chromosomes of the L. major genome $(32.8 \mathrm{Mb})$ was published, and provided a framework for future comparative genomic studies [13]. Using bioinformatic analyses, 911 RNA genes, 39 pseudogenes, and 8272 protein-coding genes were predicted. Within the latter group, only $36 \%$ can be assigned to a putative function based on sequence conservation with protein characterized in other eukaryotic organisms. Most L. major genes have orthologs in the T. brucei and T. cruzi genomes [14]. However, more than $60 \%$ of the predicted genes remain annotated as hypothetical. A major challenge lies ahead to discover whether or not these genes are expressed at any moment in the life cycle and, therefore, may be catalogued as functional genes. On the other hand, both known and putative genes lack annotated 5' and 3' untranslated regions (UTRs), and for only a few genes these regions have been experimentally determined [15]. In
Leishmania, and related trypanosomatids, these flanking regions (largely the 3'-UTRs) have been involved in regulating the steady-state level and translational status of specific mRNAs along the cell cycle and in the different life cycle stages [10-12].

Recent advances in sequencing technologies, known as deep sequencing or next-generation sequencing (NGS), are becoming invaluable tools, among others, for reconstructing of the entire transcriptome of a given organism $[16,17]$. In this study, we employed the power of NGS on RNA analysis (RNA-seq) to provide a comprehensive characterization of the poly-A transcriptome for the promastigote stage of Leishmania major. A total of 10285 transcripts were identified, of which 1884 did not match with previously annotated genes and therefore were categorized as novel genes. In addition, the RNA-seq analysis generated valuable information on both the relative abundance of the RNAs and the structures of their corresponding genes (i.e. ORFs, and 5'- and 3'-UTRs).

\section{Methods}

\section{Leishmania culture and RNA isolation}

Promastigotes of L. major Friedlin strain (MHOM/IL/80/ Friedlin; clone V1) were cultured at $26^{\circ} \mathrm{C}$ in RPMI medium supplemented with $10 \%$ fetal bovine serum, 100 $\mathrm{U} / \mathrm{ml}$ penicillin $\mathrm{G}$ and $0.1 \mathrm{mg} / \mathrm{mL}$ streptomycin sulphate. Promastigotes were grown to mid log phase by seeding cultures at $1 \times 10^{6}$ cells $/ \mathrm{mL}$, and collected for RNA isolation when the culture density reached $6.1 \times 10^{6}$ cells $/ \mathrm{mL}$ (mid-logarithmic phase of growth). Total RNA was isolated using the Aurum ${ }^{\mathrm{TM}}$ Total RNA Mini Kit (Biorad), and treated with RNAse-free DNAse I. RNA samples were quantified by absorbance at $260 \mathrm{~nm}$ using the Nanodrop ND-1000 (Thermo Scientific), all samples showed an $\mathrm{A}_{260} / \mathrm{A}_{280}$ ratio higher than 2.0. In addition, RNA integrity was checked in a bioanalyzer (Agilent 2100).

\section{RNA-seq and data processing}

RNA-seq was performed at the Massive Sequencing Platform of Cantoblanco (CSIC-PCM, Madrid, Spain). Standard libraries for massive sequencing were generated using the TruSeq RNA Sample Prep Kit (Illumina). Briefly, poly- $\mathrm{A}^{+}$RNA was selected by oligo-dT chromatography, and RNA fragmentation was achieved using divalent cations under elevated temperature. Afterwards, these fragments were used to generate a cDNA library, and cDNA fragments corresponding in size to about 300$400 \mathrm{bp}$ were selected on an agarose gel. Two cDNA libraries were constructed: first strand synthesis of one of them was initiated with only random hexadeoxynucleotide primers (Illumina standard protocol); however, for the first strand synthesis of the second library, we introduced as an additional component the 5 ' $-\mathrm{T}_{15} \mathrm{VN}-3$ ' oligonucleotide together with the random hexamer primers present in the 
kit. Afterwards, the second strand of the cDNA was synthesized. The cDNA ends were repaired and adenylated, subsequently adapters were added at both ends. Finally, the library was enriched in ligated fragments by limited PCR amplification. Sequencing was carried out in a GAIIx Illumina system. Each library was sequenced in two separated lines. Single reads of 75 nucleotides were obtained, and raw reads were subject to quality-filtered using the standard Illumina process and analyzed using FASTQC tool [18]. Reads were mapped to the last assembled version of $L$. major genome, obtained from the Sanger Institute (ftp://ftp.sanger.ac.uk/pub/pathogens/Leishmania/major/ V6_211210/), using Bowtie [19]. In the alignment of reads, a maximal of three mismatches was allowed within the whole read (aligner $\mathrm{V}$ mode). Nevertheless, in order to select the best alignment in terms of number of mismatches, the option "-best" was used. Also, the option "-k1" was elected, i.e. if in the course of the search Bowtie found 2 (or more) possible alignments for a given read, the program selected one of the alignments at random. We analyzed different alignment conditions in terms of multi-hits in order to obtain the best and accurate results from our data. Allowing up to 10 multi-hits for a single read, the main differences with the transcripts assembled with no multi-hits restriction were found at gene-tandem repeat regions. In those regions the assembled transcripts were reduced to the UTRs, losing the coding regions. Therefore, no restriction in the number of multi-hits was introduced, except for SL-containing reads, in which reads mapping to more than 10 sites were excluded for further analysis. Finally, mapped reads were assembled into transcripts using Cufflinks [20].

\section{Identification of trans-splicing and polyadenylation sites}

Among the non-aligned reads, a search for reads containing 8 (or more) nucleotides identical to the 3'-end of the SL sequence (AACTAACGCT ATATAAGTAT CAGTTTCTGT ACTTTATTG) was performed using a custom Perl script. No mismatches were allowed. Afterwards, the SL-matching nucleotides were stripped from the reads and the remaining sequence was used to map the position of the trans-splicing site in the reference genome. Similarly, reads spanning potential polyadenylation sites were extracted from the non-aligned sequences by an in house Perl script, which finds reads with A-runs (higher than $5 \mathrm{nu}$ cleotides in length) located at an end of the read sequence. These reads were mapped back to the reference genome.

\section{Additional sequencing analysis tools}

Samtools software [21] was used to interconvert alignment formats, and to assign the annotated genes to transcripts generated from Seqdata, a local version of Blastx program [22]. The IGV browser was used [23] for visualization of mapped reads and assembling of transcripts to its genome context. Consensus sequences were analyzed using a local version of WebLogo tool [24]. BLAST searches for sequence homologies were performed in the following databases: GeneDB [25], TritrypDB [26] and GenBank at the NCBI [27].

\section{Results and discussion}

\section{RNA-seq data and delineation of transcripts}

RNA isolated from an axenic culture of $L$. major promastigotes (Friedlin strain, clone V1) was sequenced, after poly $(\mathrm{A})+$ selection, on an Illumina GAII platform generating a total of 14 656121 sequence reads (75-nt long). RNA-seq data from this study have been submitted to the EBI-ENA Sequence Read Archive (SRA) under accession number ERP002077. Allowing up to three mismatches, 14027356 reads (95.71\%) were aligned to the reference $L$. major Friedlin genome [13]. After initial assembling, it was possible to define a total of 6937 transcripts; a number lower than the 8272 protein-coding genes previously predicted to exist in the L. major genome [13]. However, as shown in Figure 1, this difference was not derived from a low coverage of RNA-seq data. Instead, the transcript assembly indicated that most of the Leishmania genome seems to be transcribed, and many assembled transcripts contain two or more annotated coding-genes (Figure 1). In fact, the genome coverage of the RNA-seq reads generated in this study was around $90.75 \%$, even though reads for tRNAs, SL-RNAs and other small RNAs were not obtained. Several possibilities may be envisioned to accommodate this observation. First, existence of stable polycistronic transcripts; however, to date there are not descriptions of mature polycistronic transcripts in Leishmania. Nevertheless, the existence of a functional bicistronic transcript has been demonstrated in T. cruzi [28]. A second possibility is that some RNA processing intermediates with larger half-life may be represented in the RNA-seq reads. This hypothesis is very plausible as there are many reports describing processing intermediates that are clearly detected by Northern blot analysis. For example, at least 10 stable cytoplasmic poly(A) + RNAs, ranging in size from 1.7 to $13 \mathrm{~kb}$ and related to the $3.2-\mathrm{kb}$ DHFR-TS mRNA have been observed in antifolateresistant Leishmania promastigotes [29]. In other studies, polycistronic intermediates were demonstrated using a combination of genic and intergenic probes [30]. Third, antisense transcription might be contributing to create polycistronic transcript, since RNA-Seq data were derived from non-oriented, unidirectional sequencing of RNA molecules. There are several reports describing the existence of antisense transcription in Leishmania. For example Monnerat and co-workers [31], analyzing the transcriptional activity of a $30-\mathrm{Kb}$ region from $L$. major chromosome 27, found that while the non-coding strand 


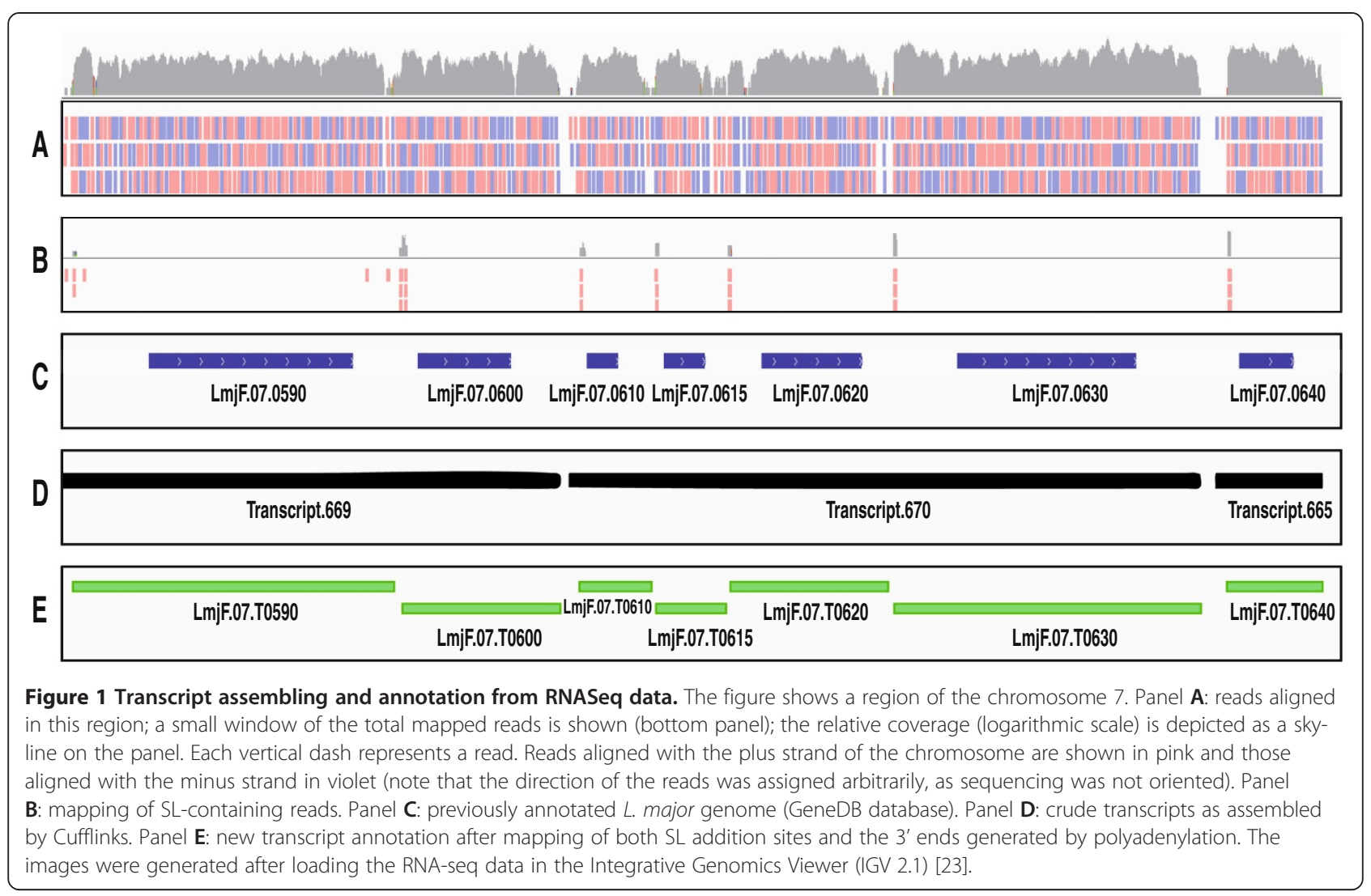

generally appears to be transcribed at levels close to background, several regions appeared to be transcribed at significant levels, albeit still substantially lower than the coding strand. A fourth possibility, a background derived from sequencing of contaminating DNA, may be discarded, since there are many intergenic regions from which there were no reads (see gaps without reads on Figure 1A). However, if DNA contamination were present in the RNA samples, reads should be mapped to all chromosomal locations.

In order to further delineate Leishmania transcripts, we took advantage of the expected addition of the 39nucleotide long mini-exon sequence at the 5 '-end of all Leishmania mRNAs [32,33]. Thus, we searched among the non-aligned reads ( $628765 ; 4.29 \%$ of total reads) for sequences containing at the 5'-end eight (or more) nucleotides identical to the 3'-end of the mini-exon sequence. A total of 188398 sequence reads met these criteria. After trimming the mini-exon sequences, these reads were aligned to the L. major genome (Figure 1B) and, as a result, 22592 different mini-exon addition sites were defined.

Interestingly, only 44, of the 188398 reads containing SL sequences, were mapped in antisense orientation (related to the coding strand), suggesting that trans-splicing occurs almost exclusively in sense transcripts and that antisense transcripts (if produced at meaningful levels) should not be processed by the addition of mini-exon sequences. In a recent published work [8], the authors describe the role that base J plays in termination of RNAP II transcription in L. tarentolae, mentioning that the vast majority of SL-containing reads were restricted to the coding strand. Proper transcription termination and avoidance of readthrough of transcriptional stops seemed to be vital for Leishmania [8].

As illustrated in Figure 1, most of the SL-containing reads mapped at expected locations, i.e. upstream of annotated genes and a significant number of reads were found for each putative splicing acceptor site (considering both main and alternative sites). However, exceptions for this rule were also found. Thus, from time to time, single reads containing SL sequences were mapped at unexpected positions, such as coding sequences or 3'-UTRs. Furthermore, the position of those reads was not accompanied by a breakdown in the reads density as occurs for the rest of SL addition sites. A plausible interpretation for these findings is that the transsplicing machinery generates a low, but detectable number of events in which the mini-exon is misplaced. Keeping in mind this idea, we excluded in the transcript defining process those mini-exon addition sites that were defined by a sole read and located at unexpected positions.

Finally, using as criterion for defining the 5' end of a transcript the location of a SL addition site, most of the 
polycistronic transcripts obtained after the initial assembling could be split up, giving a total number of 10285 transcripts (Table 1). Only 73 of these transcripts remained as polycistronic, with 72 bicistronics and one tetracistronic (LmjF.30.T1460-1470-1480-1490). It would be interesting to analyze whether these bicistronic

Table 1 Transcriptome of Leishmania major promastigotes

\begin{tabular}{|c|c|c|c|c|}
\hline Chromosome & $\begin{array}{l}\text { Number of } \\
\text { transcripts }\end{array}$ & $\begin{array}{l}\text { Bicistronic } \\
\text { transcripts }\end{array}$ & $\begin{array}{c}\text { Non- } \\
\text { annotated } \\
\text { genes }\end{array}$ & $\begin{array}{c}\text { Mis- } \\
\text { annotated } \\
\text { genes }(*)\end{array}$ \\
\hline 1 & 92 & & 7 & 1 \\
\hline 2 & 93 & 2 & 19 & $9(1)$ \\
\hline 3 & 110 & & 13 & 9 \\
\hline 4 & 140 & 1 & 11 & $14(1)$ \\
\hline 5 & 146 & 1 & 22 & $4(2)$ \\
\hline 6 & 154 & & 19 & $7(3)$ \\
\hline 7 & 158 & & 28 & $6(7)$ \\
\hline 8 & 171 & 1 & 36 & $3(1)$ \\
\hline 9 & 189 & 1 & 21 & $6(3)$ \\
\hline 10 & 175 & 4 & 32 & $5(4)$ \\
\hline 11 & 171 & 1 & 34 & $1(2)$ \\
\hline 12 & 183 & & 41 & $12(1)$ \\
\hline 13 & 207 & 1 & 38 & $7(5)$ \\
\hline 14 & 194 & & 35 & $5(2)$ \\
\hline 15 & 196 & 2 & 28 & $11(2)$ \\
\hline 16 & 213 & 1 & 37 & $8(3)$ \\
\hline 17 & 211 & 1 & 52 & 5 \\
\hline 18 & 242 & & 70 & $5(2)$ \\
\hline 19 & 216 & & 38 & $4(1)$ \\
\hline 20 & 215 & 5 & 40 & 9 \\
\hline 21 & 264 & & 37 & $11(2)$ \\
\hline 22 & 214 & & 45 & $7(1)$ \\
\hline 23 & 253 & 3 & 53 & $8(3)$ \\
\hline 24 & 286 & 1 & 48 & $14(1)$ \\
\hline 25 & 302 & 4 & 50 & $22(1)$ \\
\hline 26 & 330 & & 51 & $10(2)$ \\
\hline 27 & 340 & 2 & 59 & $10(1)$ \\
\hline 28 & 403 & 1 & 84 & $13(2)$ \\
\hline 29 & 372 & 3 & 77 & 10 \\
\hline 30 & 465 & $2^{a}$ & 69 & $11(3)$ \\
\hline 31 & 463 & 5 & 126 & $51(12)$ \\
\hline 32 & 509 & 6 & 85 & $25(4)$ \\
\hline 33 & 492 & 5 & 115 & $13(4)$ \\
\hline 34 & 570 & 6 & 88 & $25(1)$ \\
\hline 35 & 673 & 5 & 133 & $9(10)$ \\
\hline 36 & 873 & 9 & 143 & $40(7)$ \\
\hline Genome & 10285 & 73 & 1884 & $410(94)$ \\
\hline
\end{tabular}

*In brackets is indicated the number of genes that might be truncated by addition of SL in secondary trans-splicing sites.

${ }^{a}$ Transcript LmjF.30.T1460-1470-1480-1490 is tetracistronic. transcripts really exist or they are only evidencing current annotation deficiencies in the L. major database. A detailed list of the L. major transcriptome is provided as an Additional file 1. Transcripts were named using the systematic identifiers for the annotated genes [13], and were interdigitated numbers to name the new transcripts. In order to distinguish between transcript and genes, a $\mathrm{T}$ preceding the transcript number was included. By way of example, Figure 1 (panel C) shows the previously annotated genes existing in a region of chromosome 7 , and in panel $\mathrm{E}$ are shown the new transcripts (and their names) mapped at that chromosomal region.

With the sole exception of genes LmjF.02.0400, LmjF.09.0690, LmjF27.0280, LmjF33.1760, LmjF35.2600 and LmjF35.2610, transcripts were found for all the currently annotated genes at GeneDB database [25]. These six genes code for hypotethical proteins, but, at least, gene LmjF35.2610 seems to be encoding a protein since the predicted amino acid sequence contains a region with similarity to ubiquitin and also an AT hook, DNA-binding motif; furthermore, the gene is present in other Leishmania species [26]. Thus, the lack of expression of these genes, and in particular of LmjF35.2610, in $L$. major promastigotes is a finding that would merit further studies.

Interestingly, 1884 new transcripts were found spanning genomic regions lacking annotated genes; hence, they were categorized as non-annotated genes (Table 1). These findings suggest that the gene content of $L$. major would be approximately $20 \%$ higher than previously believed [13]. Similar results have been reported after determining the T. brucei transcriptome by RNA-seq [34]. Nevertheless, it is likely that many of these new transcripts may have roles other than protein-coding function; some may even be merely processing products resulting from the unusual polycistronic gene organization and processing of the Leishmania genome. In this regard, non-coding transcripts, derived from intercoding regions of T. brucei VSG genes, were found to be trans-spliced, polyadenylated and present in polyribosomes [35]. Therefore, the new transcripts described in this work might be considered non-coding (nc) RNAs until shown to be otherwise.

Concerning the 5'-end mapping, we have shown that 410 annotated genes are mis-predicted, regarding the translation start codons, as splice acceptor sites were found exclusively downstream of the previously assigned ATG. An example for a clear mis-annotation is shown in Figure 2. Thus, three SL addition sites were found to exist in the middle of the ORF currently annotated as LmjF.04.0860; however, no SL addition sites were found at the 5 ' end of the annotated gene and no reads were mapped at the region coding for the $\mathrm{N}$-terminal moiety of LmjF.04.0860. Translation from the nearest ATG codon found after the main SL addition site gives a protein corresponding to the last 240 amino acids of the annotated 
LmjF.04.0860 protein (Figure 2B). Interestingly, the new protein is similar in size and sequence to that encoded by the gene Tb927.9.8290, which has an authentic annotation in the T. brucei databases (Figure 2C). In the GeneDB database this hypothetical protein is categorized as conserved, since it is also encoded in the genomes of T. cruzi and other Leishmania species. As a structural feature, the protein contains the domain SSF55129, which is typical of the ribosomal protein L30p/L7e superfamily. In summary, these data support the conclusion that mature mRNAs containing the LmjF.04.0860 ORF, as annotated in the GeneDB database, do not exist.

On the other hand, for 94 annotated genes, alternative splice addition sites were mapped into the ORF, suggesting that different proteins might be generated from a single gene. In this regard, there is a documented case of alternative trans-splicing in the T. cruzi LYT1 gene, in which the different maturation of the mRNA leads to the expression of protein isoforms showing different compartmental and functional properties [36]. Overall, our transcriptomic study has uncovered that the current annotation of the L. major genome had clear limitations that are corrected by the data reported in this work.

\section{Determination of RNA levels from RNA-seq data}

RNA-seq is an accurate method for quantifying transcript levels. The strength of this method is that it produces digital counts of transcript abundance, in contrast to the analog-style signals obtained from fluorescent dye-based microarrays. This technique has been validated by several studies and found to be highly reproducible, with very little technical variability and can measure mRNA levels over several orders of magnitude [37,38]. A useful parameter is FPKM (fragments or reads- per kilobase of transcript per million mapped reads), which reflects the abundance of a transcript in the sample by normalizing for RNA length and for the total read number in the measurement [39]. Thus, the presence and abundance of a given RNA can be calculated and subsequently compared with the amount in any other sequenced sample, now or in the future.

Table 2 contains a list with the 50 most abundant transcripts detected in promastigotes of $L$. major. Two of the top three on the list are transcripts corresponding to the heat shock protein 70 (HSP70); this finding is not unexpected taking into account that this protein make up $2.1 \%$ of the total protein in unstressed Leishmania promastigotes [40]. Additionally, the fact that 20 out of 50 correspond to transcripts encoding ribosomal proteins might indicate that a direct correlation between transcript levels and protein abundance would be a general rule in Leishmania. Another conspicuous observation is that 18 out of the 50 most abundant transcripts derive from genes located on chromosome 35 . However, at first glance, these genes do not seem to be concentrated at specific regions of the chromosome; rather they seem to be randomly distributed. Other abundant transcripts are those encoding nucleoside transporters, histone H4, peptidases, cyclophilin, LACK and tubulins (Table 2). Two abundant transcripts, LmjF.35.T2220 and LmjF.35. T2210, encode KMP-11, a protein found tightly associated with lipophosphoglycan, the major cell surface glycoconjugate of Leishmania promastigotes [41]. In addition, there are other transcripts encoding for hypothetical proteins that are expected to be abundant ones. Thus, transcripts LmjF.31.T0900 (the fifth on the list) would be coding for a hypothetical, small protein (79 amino acids), annotated as LmjF31.0900, which is also present in the genomes of related trypanosomatids. Interestingly, this transcript was identified in previous studies to be both abundant and differentially expressed in promastigotes by using oligonucleotide microarrays [42], and one of the most abundant transcripts in metacyclic $L$. major grown in culture by using SAGE methodologies [43]. Another abundant transcript, LmjF.31. T0966, located at a region lacking annotated genes contains high sequence identity with gene LmjF31.0900. The structural relationship between both transcripts (LmjF.31.T0900 and LmjF.31.T0966) and the functional role of the encoded protein (LmjF31.0900) are two aspects that merit further studies. Transcript LmjF.36.T3620, containing the annotated LmjF36.3620 gene and coding for a hypothetical protein, was also found among the most abundant transcripts in L. major promastigotes [42] and metacyclics forms [43].

On the other hand, five transcripts (LmjF.19.T0983, LmjF.20.T1285, LmjF.31.T0964, LmjF.31.T0895, and LmjF.35. T4191), among the most abundant in L. major promastigotes (Table 2), do not contain previously annotated genes. The sequence of transcript LmjF.19.T0983 was found to be conserved in the genomes of different Leishmania species (L. braziliensis, L. donovani, L. mexicana and L. infantum) but conserved sequences were not detected in the genomes of related trypanosomatids (i.e. T. brucei and T. cruzi). Interestingly, a cDNA (named DRS-2) derived from this transcript was previously described in L. major as an mRNA whose expression increases during metacyclogenesis [15]. Similarly, sequences homologous to transcript LmjF.31. T0964 were found in the genomes of all Leishmania species sequenced to date, but absent in the genus Trypanosoma. The sequences of transcripts LmjF.20.T1285, LmjF.31.T0895 and LmjF.35.T4191 were found to be well conserved in the genomes of L. donovani, L. mexicana and $L$. infantum, but seemed to be absent from the $L$. braziliensis genome. It is clear that a challenge for the future will be to understand the nature (coding or not) of these transcripts, and certainly for the additional 1879 new transcripts that have been described in this work (Table 1).

Tandemly repeated, multi-copy gene loci are frequent in the Leishmania genome [11]. In fact, the list shown in 
A
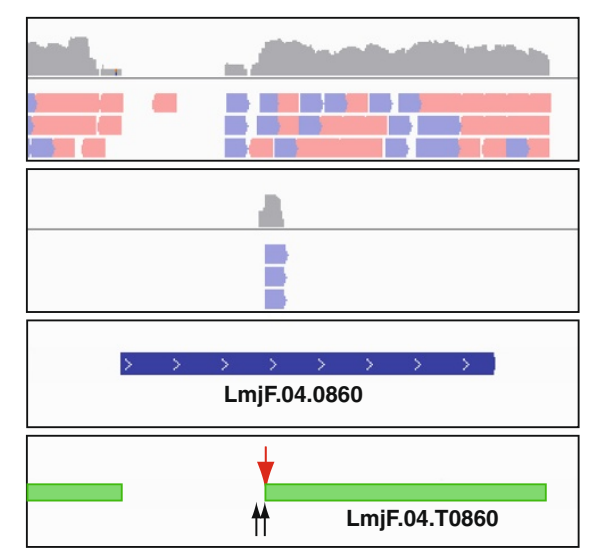

\section{Reads mapping}

\section{SL-Reads}

\section{GeneDB annotation}

Transcript annotation

B

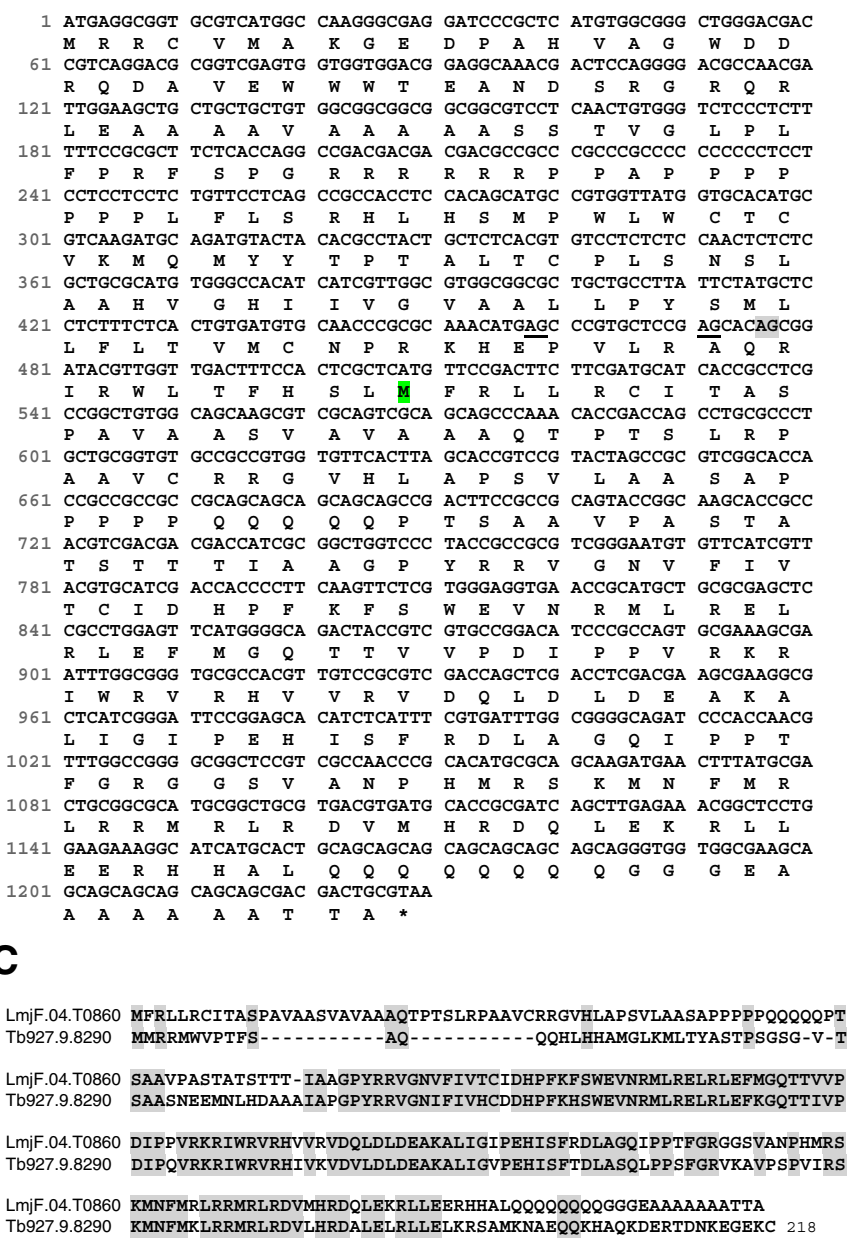

Figure 2 Mis-annotation of LmjF04.0860 gene. (A) Upper panels show the mapping of RNA-seq reads (either total or SL-containing reads) in the genomic region containing the annotated LmjF04.0860 gene; the bottom panel contains the transcripts delimited in this region. Arrows indicate SL addition sites, the red arrow points at the main SL addition site. Reads aligned with the plus strand of the chromosome are shown in violet and those aligned with the minus strand in pink (note that the direction of the reads was a consequence of sequencing process, as sequencing was not oriented). (B) Nucleotide sequence (and predicted amino acid sequence) of the LmjF04.0860 gene as annotated in the GeneDB database [25]. Shaded in gray is shown the position of the main AG dinucleotide used for trans-splicing in the LmjF.04.T0860 transcripts, and underlined are those AG dinucleotide representing alternative SL addition sites. Shaded in green it is shown the first ATG found downstream the SL addition sites. (C) Alignment between the protein predicted in the LmjF.04.T0860 transcript and the Tb927.9.8290 protein annotated in the T. brucei GeneDB database [25]. Identical amino acids are shaded in gray. 
Table 2 The $\mathbf{5 0}$ most abundant transcripts in L. major promastigotes

\begin{tabular}{|c|c|c|c|}
\hline Transcript & Gene $^{a}$ & FPKM $^{\mathrm{b}}( \pm \mathrm{SD})$ & Remarks $^{c}$ \\
\hline LmjF.28.T2770 & LmjF28.2770 & $1357.39 \pm 5.12$ & heat-shock protein (HSP70; gene HSP70-II) \\
\hline LmjF.35.T0240 & LmjF35.0240 & $1034.68 \pm 12.39$ & ribosomal protein $L 30$ \\
\hline LmjF.28.T2780 & LmjF28.2780 & $987.24 \pm 4.45$ & heat-shock protein hsp70 (HSP70; gene HSP70-I) \\
\hline LmjF.36.T1940 & LmjF36.1940 & $952.68 \pm 4.37$ & inosine-guanosine transporter (NT2) \\
\hline LmjF.31.T0900 & LmjF31.0900 & $809.07 \pm 7.12$ & hypothetical protein, conserved \\
\hline LmjF.28.T2205 & LmjF28.2205 & $792.33 \pm 8.16$ & ribosomal protein $\mathbf{S} 29$ \\
\hline LmjF.35.T2220 & LmjF35.2220 & $780.12 \pm 5.99$ & kinetoplastid membrane protein-11 (KMP11) \\
\hline LmjF.19.T0983 & Non-annotated & $674.85 \pm 5.26$ & - \\
\hline LmjF.35.T0600 & LmjF35.0600 & $672.00 \pm 5.81$ & ribosomal protein L18a \\
\hline LmjF.06.T0010 & LmjF06.0010 & $666.85 \pm 8.31$ & histone $\mathrm{H} 4$ \\
\hline LmjF.35.T3800 & LmjF35.3800 & $617.33 \pm 7.36$ & ribosomal protein $L 23$ \\
\hline LmjF.36.T3620 & LmjF36.3620 & $616.48 \pm 5.07$ & hypothetical protein, conserved \\
\hline LmjF.35.T2210 & LmjF35.2210 & $603.26 \pm 4.69$ & kinetoplastid membrane protein-11 (KMP11) \\
\hline LmjF.28.T2460 & LmjF28.2460 & $596.61 \pm 6.27$ & ribosomal protein $\mathbf{S} 29$ \\
\hline LmjF.20.T1285 & Non-annotated & $560.05 \pm 5.48$ & - \\
\hline LmjF.31.T0964 & Non-annotated & $539.44 \pm 5.07$ & - \\
\hline LmjF.31.T0895 & Non-annotated & $524.19 \pm 10.51$ & - \\
\hline LmjF.35.T3290 & LmjF35.3290 & $514.13 \pm 5.67$ & ribosomal protein $\mathrm{L} 31$ \\
\hline LmjF.13.T0570 & LmjF13.0570 & $496.01 \pm 5.02$ & ribosomal protein $\mathrm{S} 12$ \\
\hline LmjF.35.T3790 & LmjF35.3790 & $493.33 \pm 8.18$ & ribosomal protein $\mathrm{L} 23$ \\
\hline LmjF.35.T4191 & Non-annotated & $490.94 \pm 5.15$ & - \\
\hline LmjF.35.T3760 & LmjF35.3760 & $483.03 \pm 7.04$ & ribosomal protein L27A/L29 \\
\hline LmjF.30.T3340 & LmjF30.3340 & $482.87 \pm 5.89$ & ribosomal protein L9 \\
\hline LmjF.35.T2050 & LmjF35.2050 & $464.76 \pm 5.1$ & ribosomal protein $\mathrm{L} 32$ \\
\hline LmjF.08.T0640 & LmjF08.0640 & $452.92 \pm 3.18$ & hypothetical protein \\
\hline LmjF.14.T0850 & LmjF14.0850 & $451.18 \pm 3.65$ & calpain-like cysteine peptidase \\
\hline LmjF.35.T1910 & LmjF35.1910 & $448.56 \pm 5.89$ & ribosomal protein L15 \\
\hline LmjF.35.T0420 & LmjF35.0420 & $446.58 \pm 5.12$ & ribosomal protein S3A \\
\hline LmjF.35.T1920 & LmjF35.1920 & $446.57 \pm 9.14$ & ribosomal protein L36 \\
\hline LmjF.25.T0910 & LmjF25.0910 & $436.47 \pm 3.61$ & cyclophilin a \\
\hline LmjF.35.T3780 & LmjF35.3780 & $427.63 \pm 4.95$ & ribosomal protein L27A/L29 \\
\hline LmjF.28.T2740 & LmjF28.2740 & $426.82 \pm 4.82$ & activated protein kinase $\mathrm{c}$ receptor \\
\hline LmjF.13.T0450 & LmjF13.0450 & $425.12 \pm 4.7$ & hypothetical protein, conserved \\
\hline LmjF.20.T1280 & LmjF20.1280 & $424.16 \pm 3.22$ & small myristoylated protein 4 \\
\hline LmjF.31.T0966 & Non-annotated $^{d}$ & $419.44 \pm 8.52$ & hypothetical protein, conserved \\
\hline LmjF.28.T2750 & LmjF28.2750 & $414.41 \pm 4.15$ & activated protein kinase $\mathrm{c}$ receptor \\
\hline LmjF.31.T1170 & LmjF31.1170 & $414.01 \pm 3.8$ & hypothetical protein \\
\hline LmjF.35.T0410 & LmjF35.0410 & $411.95 \pm 4.52$ & ribosomal protein S3A \\
\hline LmjF.15.T1240 & LmjF15.1240 & $410.71 \pm 3.38$ & nucleoside transporter 1 \\
\hline LmjF.24.T2230 & LmjF24.2230 & $409.67 \pm 3.11$ & hypothetical predicted multi-pass transmembrane protein \\
\hline LmjF.35.T3280 & LmjF35.3280 & $406.24 \pm 5.44$ & ribosomal protein L31 \\
\hline LmjF.35.T0400 & LmjF35.0400 & $403.61 \pm 4.43$ & ribosomal protein S3A \\
\hline LmjF.24.T1280 & LmjF24.1280 & $403.46 \pm 3.63$ & amastin-like surface protein \\
\hline LmjF.13.T0370 & LmjF13.0370 & $403.05 \pm 3.84$ & alpha tubulin \\
\hline LmjF.35.T1670 & LmjF35.1670 & $403.05 \pm 6.46$ & ribosomal protein L26 \\
\hline LmjF.13.T0360 & LmjF13.0360 & $403.04 \pm 3.84$ & alpha tubulin \\
\hline LmjF.13.T0350 & LmjF13.0350 & $399.2 \pm 3.82$ & alpha tubulin \\
\hline
\end{tabular}


Table $\mathbf{2}$ The $\mathbf{5 0}$ most abundant transcripts in L. major promastigotes (Continued)

\begin{tabular}{|c|c|c|c|}
\hline LmjF.13.T0380 & LmjF13.0380 & $399.06 \pm 3.82$ & alpha tubulin \\
\hline LmjF.33.T3230 & LmjF33.3230 & $396.93 \pm 7.5$ & ribosomal protein L44 \\
\hline LmjF.13.T0330 & LmjF13.0330 & $396.86 \pm 3.81$ & alpha tubulin \\
\hline
\end{tabular}

Table 2 contains several examples: genes LmjF.28.2770 and LmjF.28.2780, coding for HSP70; LmjF.35.3790 and LmjF.35.3800, coding for ribosomal protein L23; LmjF.35.2220 and LmjF.35.2210, coding for KMP-11; LmjF35.0420, LmjF35.0410 and LmjF35.0400, coding for ribosomal protein S3A; LmjF28.2740 and $L m j F 28.2750$, coding for activated protein kinase c receptor (also known as LACK in Leishmania [44]); and the five LmjF13.03300370 genes, coding for alpha tubulin. More frequently, the tandemly arranged genes have identical or highly conserved sequences in their protein coding regions. When RNA-seq reads are mapped at two or more places in the reference genome, due to sequence identity, the assembling algorithms, as that used in this study, make an equal distribution of the reads among the putative transcripts. Obviously, this fact may lead to miscalculation of the expression levels when two transcripts share conserved regions but also contain divergent ones. This can be illustrated analyzing the expression levels of transcripts derived from the HSP70 locus, i.e. transcripts LmjF.28.T2770 and LmjF.28.T2780 (Table 2). Two types of genes, HSP70$I$ and HSP70-II, are present in different Leishmania species [45]. Both types of genes have identical 5'-UTR and coding sequences, but divergent 3'-UTRs; in addition, analysis of the steady-state mRNA levels in L. infantum promastigotes indicated that transcripts derived from the HSP70-II gene are one order of magnitude more abundant than HSP70-I transcripts [46]. Indeed, according to the FPKM values shown in Table 2, the level of HSP70-II transcripts (i.e. $L m j F .28 . T 2770$ transcript) is higher than the level of HSP70-I transcripts (i.e. LmjF.28.T2780); however, the difference is lower than expected. Fortunately, the counter nature of the RNA-seq data allows defining with precision the transcription level of a particular region of a given gene, and Figure 3A shows the results of this analysis for the HSP7O locus. While, as expected, the reads mapped to the 5'-UTR + CDS of both genes are equivalent, the number of reads containing sequences belonging to the 3'UTR of HSP70-II gene (LmjF.28.2770) was 6,24 fold higher than the number of reads corresponding to the 3'UTR of HSP7O-I gene (LmjF.28.2780), even though the 3'-UTR lengths are very similar (1096 and 1084 nucleotides, respectively). These results indicate that the steadystate level for LmjF.28.T2770 (HSP70-II) transcripts is clearly higher than that for LmjF.28.T2780 (HSP70-I) transcripts, giving similar results to those determined by classical methods of mRNA expression levels [46]. Thus, this analysis demonstrated the usefulness of RNA-seq for studies of transcript abundance, and the necessity, however, of knowing and taking into account the differences in the UTR in order to determine transcript levels in a more accurate manner. To further illustrate the usefulness of RNA-seq for determining transcript abundance, we searched for another repeated genes among the more abundant transcripts listed in Table 2 . We selected those coding for ribosomal protein L23 (Figure 3B). In the L. major genome database [25], there are two tandemly linked L23 genes (LmjF.35.3790 and LmjF.35.3800). A sequence comparison showed that both genes have identical coding regions, but marked differences both in length and sequence in the 3'-UTRs. According to the number of reads obtained for each region of the genes, it was evident that transcript LmjF.35.T3800 would be more abundant than transcript LmjF.35.T3790 in the promastigote stage. Thus, after correcting by the length of the 3'-UTRs (93 nucleotides for gene LmjF.35.3790 and 299 nucleotides for gene $L m j F .35 .3800$ ), the relative steady-state level of transcript LmjF.35.T3790 was estimated to be 3-fold lower than that for transcript LmjF.35.T3800.

On the other hand, our analysis evidenced a negligible level of single nucleotide polymorphisms (SNPs) in the assembled transcripts regarding the reference genome; this is a surprising discovery taking into account that Leishmania is an aneuploid organism, in which disomic and trisomic chromosomes are more frequently observed than monosomic ones $[47,48])$. Similarly, this very low rate of heterozygosity was noted when sequencing the $L$. major genome [13] and, more recently, when Rogers and co-workers re-sequenced the $L$. major genome using the Illumina methodology [48].

\section{Heterogeneity of trans-splicing and polyadenylation sites}

The addition of a 39-nt mini-exon (or spliced leader, SL) to the 5' end of all mRNAs in Leishmania and related trypanosomatids provides the 5' cap structure for mRNA translation [32]. As noted above, we have obtained a large number of mini-exon-containing reads and this facilitated the 5 ' end-mapping for most transcripts. Furthermore, we mapped two or more SL addition sites for around 50\% of the genes, suggesting the existence of a remarkable 


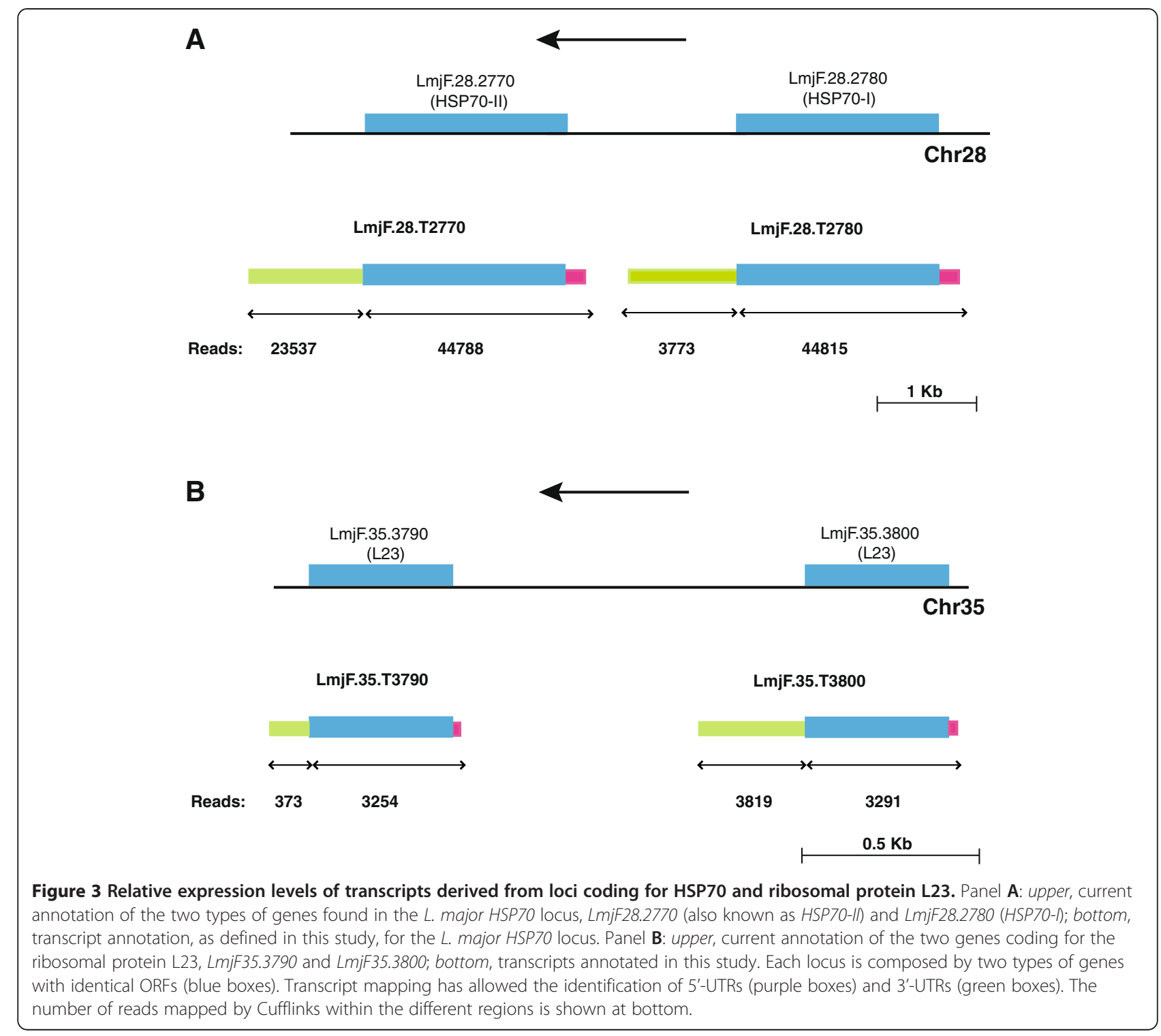

heterogeneity in the selection of the SL addition site. A similar observation has been reported in RNA-seq studies carried out in a related trypanosmatid, T. brucei $[34,49,50]$. For bioinformatics analyses, when the distance between two consecutive SL addition sites was lower than $500 \mathrm{nu}$ cleotides, they were considered alternative addition sites for a given gene. Furthermore, taking into account the numbers of reads mapping at each site, they were categorized as either main addition sites or alternative splicing sites. Thus, SL addition sites were separated into two categories: i) main SL addition sites, including unique SL addition sites or the most frequent SL addition sites when two or more sites were mapped in the same transcript; ii) alternative SL addition sites, i.e. the rest of SL addition sites in transcripts containing two of more SL addition sites. In order to avoid possible bias, SL-containing reads mapping to ten or more different genomic positions were excluded from this analysis. Most of these "multi-hit" reads mapped to the 5'-UTR of gene families containing ten or more members (Crespillo et al, manuscript in preparation). Finally, a total of $9530 \mathrm{SL}$ addition sites were classified as main sites and 4531 as alternative ones. Looking for sequence signatures associated with the SL addition sites, a compositional analysis in the \pm 40 nucleotide region surrounding the SL addition site was carried out (Figure 4). Addition of SL occurs after the well-known AG dinucleotide, even though a slight difference was observed between main and alternative sites. Thus, whereas for the main SL addition sites, the $\mathrm{A}$ and $\mathrm{G}$ frequencies were 99.82\% and $99.87 \%$, respectively, for the alternative sites the A and G frequencies were 97.26 and $99.23 \%$. Additionally, in agreement with previous analysis [51], a preference for a $\mathrm{C}$ 


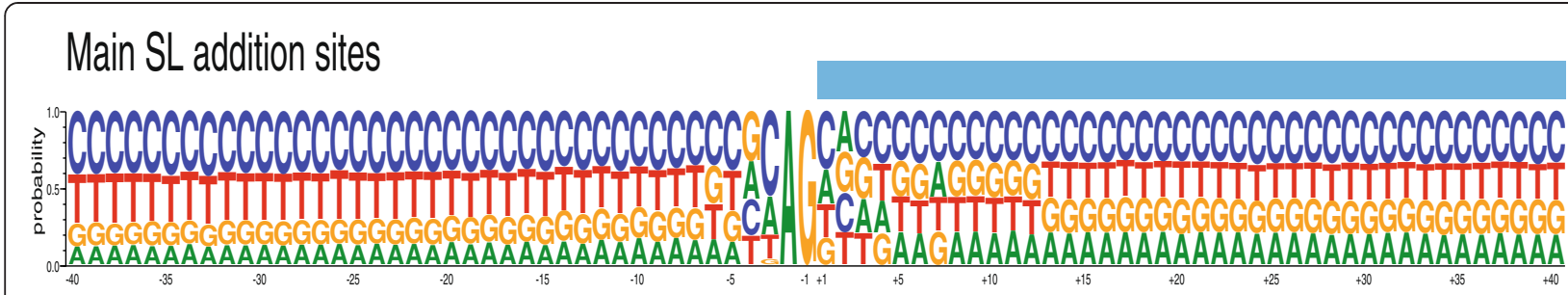

\section{Alternative SL addition sites}
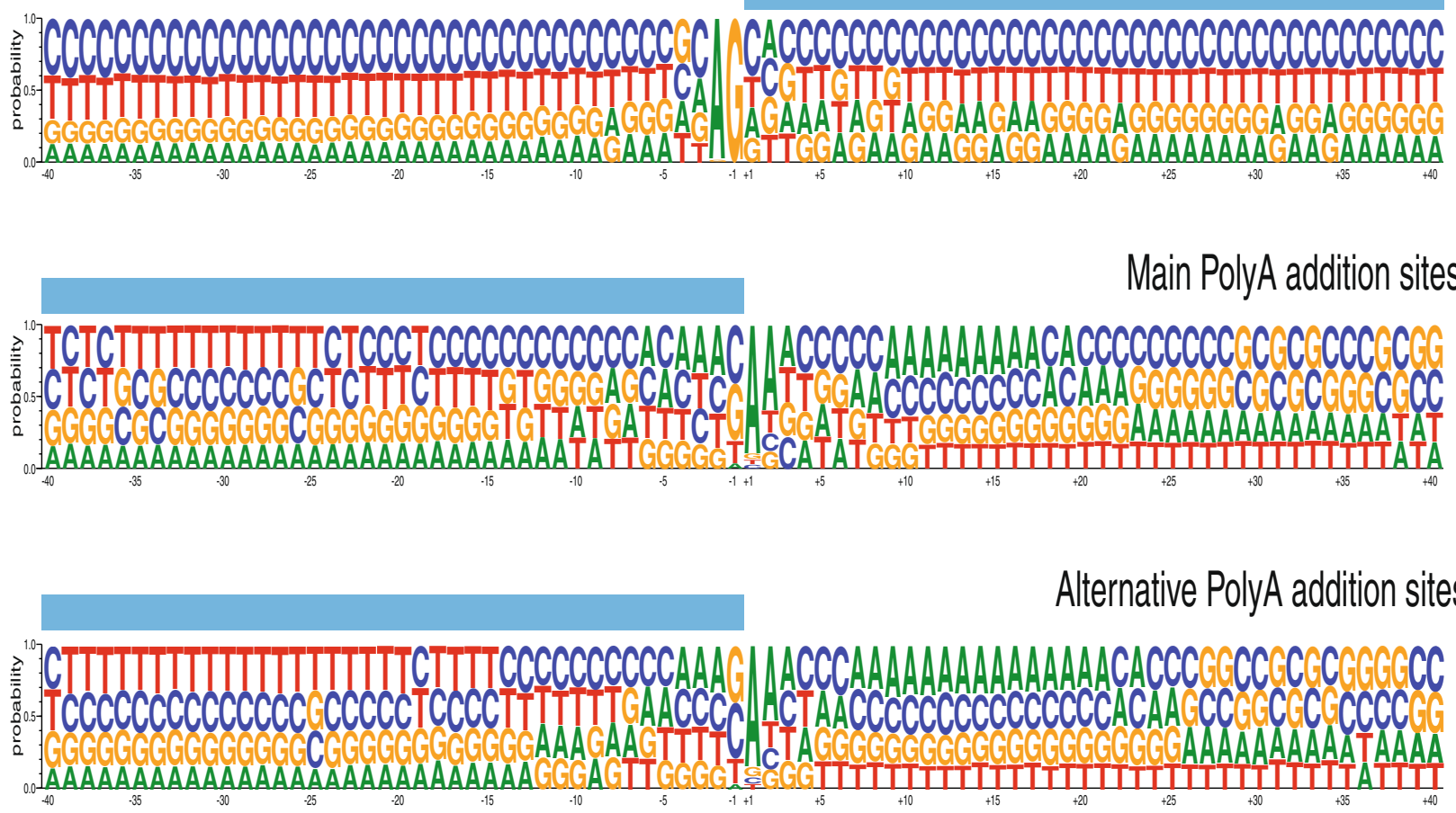

Figure 4 Nucleotide frequencies for sequences surrounding SL and polyadenylation addition sites. Panels show the compositional profiles of sequences around the main SL addition sites $(n=9530)$, alternative SL addition sites $(n=4531)$, main polyadenylation sites $(n=3178)$ and alternative polyadenylation sites $(n=1238)$.

before the AG dinucleotide was observed. Again, this preference was more marked in the main addition sites $(55.26 \%)$ than in the alternative addition sites (40.87\%). Another noticeable feature of the sequence surrounding the AG addition site is a clear richness in pyrimidine nucleotides (Figure 4). This $\mathrm{T}+\mathrm{C}$ richness is more pronounced in the upstream region: in the -40 to -21 positions the $\mathrm{T}+\mathrm{C}$ frequencies are higher than $70 \%$. Likewise, the $\mathrm{T}+\mathrm{C}$ content is higher in the regions upstream the main addition sites (71.28\%) than the alternative ones (68.68\%).

Heterogeneity in the polyadenylation sites in the Leishmania transcripts was also observed; however, the number of reads found denoting polyadenylation events was lower (7894 reads) than those mapped at the 5'end (see above), in spite that we prepared a second library in which an oligo-dT for priming was included in the cDNA reaction (see Methods section). Difficulties in the identification of polyadenylation sites were also experienced by other authors [34]. Recently, P.J. Myler and coworkers have deposited in the TriTrypDB database [26] a large number of SL- and polyadenylation sites for L. major; these new data further illustrate the complexity of trans-splicing and polyadenylation site selection in Leishmania. A comparative study between our data and those from Myler's laboratory is underway. Nevertheless, some conclusions may be drawn from the analysis of those reads mapping at the polyadenylation sites derived from our data. Polyadenylation sites were categorized as main (3178 different sites) and alternative (1238 sites). A compositional analysis of the regions surrounding the polyadenylation sites for both categories is shown in Figure 4. Searching for possible consensus sequence, we followed the consensus criteria defined by Cavener [52]: a consensus status is assigned to a single base when the frequency of a nucleotide at a certain position is greater than 
$50 \%$ and greater than twice the relative frequency of the second most frequent nucleotide; a pair of bases were assigned co-consensus status if the sum of the relative frequencies of the two nucleotides exceeded $75 \%$. The application of this rule leads to a very short consensus for the polyadenylation addition site, which may be defined as $(C / G)$ $\mathrm{AA}$; the noteworthy differences between main and alternative sites were: i) $\mathrm{C}$ is more frequent in the main sites $(40.62 \%)$ than in the alternative ones (38.77\%); ii) the frequencies for $\mathrm{A}$ residues at position 2 and 3 are higher in the main sites (88.92 and $59.1 \%$, respectively) than those found in the alternative sites (83.84 and 51.93\%, respectively). An unresolved question related to the polyadenylation consensus sequence is whether the polyadenylation occurs either before or after the AA dinucleotide. Although our data cannot elucidated this question, it is likely that the adenosines of the consensus sequences are encoded residues as it is well known that poly(A) polymerases prefer an initial adenosine residue for attachment of the poly(A) tail, and therefore the selection of the polyadenylation site would be strengthened by the presence of adenosine residues [53].

\section{Conclusions}

Sequencing and annotation of the genomes for some Leishmania species $[13,54]$ have constituted an important milestone for the study of many biological aspects of this group of parasites. The availability of these genome sequences $[25,26]$ now enables database mining and identification of different protein sets in Leishmania. This information provided new approaches to study the pattern of gene expression during differentiation and development by the use of DNA microarrays [55]. In current genome databases, the Leishmania genes lacks the definition of 5' and 3' UTRs; however, it should be noticed that recently P.J. Myler and coworkers have incorporated SL and polyA sites for most genes of $L$. major in the TriTrypDB datase [26]. The RNAseq study described here represents the first annotation of the L. major transcriptome, in which the genes have been delimited in their translated and untranslated regions. As a result, we have uncovered many cases of mis-annotated genes, and more importantly we have found 1884 new genes (previously non-annotated) in the promastigote stage. In addition, we have determined relative expression levels for each one of the 10285 transcripts detected in L. major promastigotes. In summary, the data generated by this study constitute a framework for future analysis aimed to determine differential gene expression either along the life cycle or among different Leishmania species.

\section{Additional file}

Additional file 1: An Excel file containing the complete transcriptome information for each one of the $36 \mathrm{~L}$. major chromosomes. The coordinates for each transcript are provided (Locus column), in addition to location of main (SL) and alternative SL (Alt_SL) addition sites, location of main (polyA) and alternative (Alt_polyA) polyadenylation sites. It is also indicated (GeneDB_ID) whether the transcript corresponds to a previously annotated gene (in this case the corresponding annotated gene is provided) or represents a new gene (denoted as unknown). The relative levels, expressed as FPKM, for each transcript are also provided. Finally, transcripts evidencing mis-annotated translation start codons are remarked as truncated.

\section{Competing interests}

The authors declare that they have no competing interests.

\section{Authors' contributions}

FC, BA and JMR were responsible for design and coordination of this study. AR carried out most of the bioinformatics analyses; DM, AC, RMR and JMR contributed to data analysis. JMR and BA wrote the manuscript. All the authors read and approved the final manuscript.

\section{Acknowledgements}

We are extremely grateful to Dr Julie Sheldon for English style corrections and critical reading of the manuscript. This work was funded by Ministerio de Ciencia y Tecnología [BFU2009-08986 to J.M.R., BFU 2008-03126 to B.A.], Comunidad Autonoma de Madrid (S2010/BMD-2361 to J.M.R.), and the Fondo de Investigaciones Sanitarias [ISCIII-RETIC RD06/0021/0008-FEDER to J. M.R. and R.M.R]. A.R. holded a postgraduate fellowship (FPU) from the Ministerio de Educación y Ciencia. Also, an institutional grant from Fundación Ramón Areces is acknowledged.

\section{Author details}

'Centro de Biología Molecular "Severo Ochoa" (CSIC-UAM), Universidad Autónoma de Madrid, 28049 Madrid, Spain. ${ }^{2}$ Departamento de Ciencias Biomédicas, Universidad de León, 24071 León, Spain.

Received: 4 September 2012 Accepted: 25 February 2013

Published: 4 April 2013

\section{References}

1. Desjeux P: Leishmaniasis: current situation and new perspectives. Comp Immunol Microbiol Infect Dis 2004, 27:305-318.

2. Moreira D, Lopez-Garcia P, Vickerman K: An updated view of kinetoplastid phylogeny using environmental sequences and a closer outgroup: proposal for a new classification of the class Kinetoplastea. Int J Syst Evol Microbiol 2004, 54:1861-1875.

3. Baldauf SL: The deep roots of eukaryotes. Science 2003, 300:1703-1706.

4. Myler PJ, Audleman L, DeVos T, Hixson G, Kiser P, Lemley C, Magness C, Rickel E, Sisk E, Sunkin S, et al: Leishmania major Friedlin chromosome 1 has an unusual distribution of protein-coding genes. Proc Natl Acad Sci U S A 1999, 96:2902-2906.

5. Martinez-Calvillo S, Yan S, Nguyen D, Fox M, Stuart K, Myler PJ: Transcription of Leishmania major Friedlin chromosome 1 initiates in both directions within a single region. Mol Cell 2003, 11:1291-1299.

6. Martinez-Calvillo S, Nguyen D, Stuart K, Myler PJ: Transcription initiation and termination on Leishmania major chromosome 3. Eukaryot Cell 2004, 3:506-517.

7. Thomas S, Green A, Sturm NR, Campbell DA, Myler PJ: Histone acetylations mark origins of polycistronic transcription in Leishmania major. BMC Genomics 2009, 10:152.

8. van Luenen HGAM, Farris C, Jan S, Genest PA, Tripathi P, Velds A, Kerkhoven RM, Nieuwland M, Haydock A, Ramasamy G, et al: Glucosylated hydroxymethyluracil, DNA base $\mathrm{j}$, prevents transcriptional readthrough in Leishmania. Cell 2012, 150:909-921.

9. LeBowitz JH, Smith HQ, Rusche L, Beverley SM: Coupling of poly(A) site selection and trans-splicing in Leishmania. Genes Dev 1993, 7:996-1007.

10. Fernandez-Moya SM, Estevez AM: Posttranscriptional control and the role of RNA-binding proteins in gene regulation in trypanosomatid protozoan parasites. Wiley Interdiscip Rev RNA 2010, 1:34-46.

11. Requena JM: Lights and shadows on gene organization and regulation of gene expression in Leishmania. Front Biosci 2011, 17:2069-2085.

12. Kramer S: Developmental regulation of gene expression in the absence of transcriptional control: the case of kinetoplastids. Mol Biochem Parasitol 2012, 181:61-72. 
13. Ivens AC, Peacock CS, Worthey EA, Murphy L, Aggarwal G, Berriman M, Sisk E, Rajandream MA, Adlem E, Aert R, et al: The Genome of the Kinetoplastid Parasite, Leishmania major. Science 2005, 309:436-442

14. El-Sayed NM, Myler PJ, Blandin G, Berriman M, Crabtree J, Aggarwal G, Caler E, Renauld H, Worthey EA, Hertz-Fowler C, et al: Comparative genomics of trypanosomatid parasitic protozoa. Science 2005, 309:404-409.

15. Coulson RM, Connor V, Ajioka JW: Using 3' untranslated sequences to identify differentially expressed genes in Leishmania. Gene 1997, 196:159-164.

16. Martin JA, Wang Z: Next-generation transcriptome assembly. Nat Rev Genet 2011, 12:671-682.

17. Siegel TN, Gunasekera K, Cross GAM, Ochsenreiter T: Gene expression in Trypanosoma brucei: lessons from high-throughput RNA sequencing. Trends Parasitol 2011, 27:434-441.

18. FASTQC: http://www.bioinformatics.bbsrc.ac.uk/projects/fastqc/.

19. Langmead B, Trapnell C, Pop M, Salzberg SL: Ultrafast and memoryefficient alignment of short DNA sequences to the human genome. Genome Biol 2009, 10:R25.

20. Trapnell C, Williams BA, Pertea G, Mortazavi A, Kwan G, van Baren MJ, Salzberg SL, Wold BJ, Pachter L: Transcript assembly and quantification by RNA-Seq reveals unannotated transcripts and isoform switching during cell differentiation. Nat Biotechnol 2010, 28:511-515.

21. Li H, Handsaker B, Wysoker A, Fennell T, Ruan J, Homer N, Marth G, Abecasis G, Durbin R: The Sequence Alignment/Map format and SAMtools. Bioinformatics 2009, 25:2078-2079.

22. Camacho C, Coulouris G, Avagyan V, Ma N, Papadopoulos J, Bealer K, Madden TL: BLAST+: architecture and applications. BMC Bioinformatics 2009, 10:421.

23. Robinson JT, Thorvaldsdottir H, Winckler W, Guttman M, Lander ES, Getz G, Mesirov JP: Integrative genomics viewer. Nat Biotechnol 2011, 29:24-26.

24. Crooks GE, Hon G, Chandonia JM, Brenner SE: WebLogo: a sequence logo generator. Genome Res 2004, 14:1188-1190.

25. GeneDB: www.genedb.org.

26. TritrypDB: www.tritrypdb.org

27. NCBl: www.ncbi.nlm.nih.gov.

28. Jager AV, De Gaudenzi JG, Cassola A, D'Orso I, Frasch AC: mRNA maturation by two-step trans-splicing/polyadenylation processing in trypanosomes. Proc Natl Acad Sci U S A 2007, 104:2035-2042.

29. Kapler GM, Beverley SM: Transcriptional mapping of the amplified region encoding the dihydrofolate reductase-thymidylate synthase of Leishmania major reveals a high density of transcripts, including overlapping and antisense RNAs. Mol Cell Biol 1989, 9:3959-3972.

30. Soto M, Requena JM, Alonso C: Isolation, characterization and analysis of the expression of the Leishmania ribosomal PO protein genes. Mol Biochem Parasitol 1993, 61:265-274.

31. Monnerat S, Martinez-Calvillo S, Worthey E, Myler PJ, Stuart KD, Fasel N: Genomic organization and gene expression in a chromosomal region of Leishmania major. Mol Biochem Parasitol 2004, 134:233-243.

32. Agabian N: Trans splicing of nuclear pre-mRNAs. Cell 1990, 61:1157-1160.

33. Agami R, Shapira M: Nucleotide sequence of the spliced leader RNA gene from Leishmania mexicana amazonensis. Nucleic Acids Res 1804, 1992:20.

34. Kolev NG, Franklin JB, Carmi S, Shi H, Michaeli S, Tschudi C: The transcriptome of the human pathogen Trypanosoma brucei at singlenucleotide resolution. PLoS Pathog 2010, 6:e1001090.

35. Aline RF Jr, Scholler JK, Stuart K: Transcripts from the co-transposed segment of variant surface glycoprotein genes are in Trypanosoma brucei polyribosomes. Mol Biochem Parasitol 1989, 32:169-178.

36. Benabdellah K, Gonzalez-Rey E, Gonzalez A: Alternative trans-splicing of the Trypanosoma cruzi LYT1 gene transcript results in compartmental and functional switch for the encoded protein. Mol Microbiol 2007 65:1559-1567

37. Agarwal A, Koppstein D, Rozowsky J, Sboner A, Habegger L, Hillier LW, Sasidharan R, Reinke V, Waterston RH, Gerstein M: Comparison and calibration of transcriptome data from RNA-Seq and tiling arrays. BMC Genomics 2010, 11:383.

38. Marioni JC, Mason CE, Mane SM, Stephens M, Gilad Y: RNA-seq: an assessment of technical reproducibility and comparison with gene expression arrays. Genome Res 2008, 18:1509-1517.

39. Mortazavi A, Williams BA, McCue K, Schaeffer L, Wold B: Mapping and quantifying mammalian transcriptomes by RNA-Seq. Nature methods 2008, 5:621-628.
40. Brandau S, Dresel A, Clos J: High constitutive levels of heat-shock proteins in human-pathogenic parasites of the genus Leishmania. Biochem J 1995, 310:225-232.

41. Jardim A, Funk V, Caprioli RM, Olafson RW: Isolation and structural characterization of the Leishmania donovani kinetoplastid membrane protein-11, a major immunoreactive membrane glycoprotein. Biochem $f$ 1995, 305:307-313.

42. Leifso K, Cohen-Freue G, Dogra N, Murray A, McMaster WR: Genomic and proteomic expression analysis of Leishmania promastigote and amastigote life stages: the Leishmania genome is constitutively expressed. Mol Biochem Parasitol 2007, 152:35-46.

43. Guerfali FZ, Laouini D, Guizani-Tabbane L, Ottones F, Ben-Aissa K, Benkahla A, Manchon L, Piquemal D, Smandi S, Mghirbi O, et al: Simultaneous gene expression profiling in human macrophages infected with Leishmania major parasites using SAGE. BMC Genomics 2008, 9:238.

44. Mougneau E, Altare F, Wakil AE, Zheng S, Coppola T, Wang ZE, Waldmann R, Locksley RM, Glaichenhaus N: Expression cloning of a protective Leishmania antigen. Science 1995, 268:563-566.

45. Folgueira C, Cañavate C, Chicharro C, Requena JM: Genomic organization and expression of the HSP70 locus in New and Old World Leishmania species. Parasitology 2007, 134:369-377.

46. Quijada L, Soto M, Alonso C, Requena JM: Analysis of post-transcriptional regulation operating on transcription products of the tandemly linked Leishmania infantum hsp70 genes. J Biol Chem 1997, 272:4493-4499.

47. Sterkers Y, Lachaud L, Crobu L, Bastien P, Pages M: FISH analysis reveals aneuploidy and continual generation of chromosomal mosaicism in Leishmania major. Cell Microbiol 2011, 13:274-283.

48. Rogers MB, Hilley JD, Dickens NJ, Wilkes J, Bates PA, Depledge DP, Harris D, Her $Y$, Herzyk $P$, Imamura $H$, et al: Chromosome and gene copy number variation allow major structural change between species and strains of Leishmania. Genome Res 2011, 21:2129-2142.

49. Siegel TN, Hekstra DR, Wang X, Dewell S, Cross GAM: Genome-wide analysis of mRNA abundance in two life-cycle stages of Trypanosoma brucei and identification of splicing and polyadenylation sites. Nucleic Acids Res 2010, 38:4946-4957.

50. Nilsson D, Gunasekera K, Mani J, Osteras M, Farinelli L, Baerlocher L, Roditi I, Ochsenreiter T: Spliced leader trapping reveals widespread alternative splicing patterns in the highly dynamic transcriptome of Trypanosoma brucei. PLoS Pathog 2010, 6:e1001037.

51. Requena JM, Quijada L, Soto M, Alonso C: Conserved nucleotides surrounding the trans-splicing acceptor site and the translation initiation codon in Leishmania genes. Exp Parasitol 2003, 103:78-81.

52. Cavener DR: Comparison of the consensus sequence flanking translational start sites in Drosophila and vertebrates. Nucleic Acids Res 1987, 15:1353-1361.

53. Wahle E, Keller W: The biochemistry of 3'-end cleavage and polyadenylation of messenger RNA precursors. Annu Rev Biochem 1992, 61:419-440.

54. Peacock CS, Seeger K, Harris D, Murphy L, Ruiz JC, Quail MA, Peters N, Adlem E, Tivey A, Aslett M, et al: Comparative genomic analysis of three Leishmania species that cause diverse human disease. Nat Genet 2007, 39:839-847.

55. Cohen-Freue G, Holzer TR, Forney JD, McMaster WR: Global gene expression in Leishmania. Int J Parasitol 2007, 37:1077-1086.

doi:10.1186/1471-2164-14-223

Cite this article as: Rastrojo et al:: The transcriptome of Leishmania major in the axenic promastigote stage: transcript annotation and relative expression levels by RNA-seq. BMC Genomics 2013 14:223. 